\section{Detecção de maus-tratos contra a criança: oportunidades perdidas em serviços de emergência na cidade do Rio de Janeiro, Brasil}

\author{
Detection of child abuse: missed opportunities in \\ emergency rooms in Rio de Janeiro, Brazil
}

\author{
${ }^{1}$ Faculdade de Ciências \\ Médicas, Universidade do \\ Estado do Rio de Janeiro, Rio \\ de Janeiro, Brasil. \\ 2 Instituto de Medicina \\ Social, Universidade do \\ Estado do Rio de Janeiro, Rio \\ de Janeiro, Brasil. \\ Correspondência \\ A. T. M. S. Moura \\ Departamento de Pediatria, \\ Faculdade de Ciências \\ Médicas, Universidade do \\ Estado do Rio de Janeiro. \\ Boulevard 28 de Setembro 77 , \\ Rio de Janeiro, $R J$ \\ 20551-900, Brasil. \\ annatereza.smoura@terra.com.br
}

\section{Abstract}

Emergency rooms require special consideration since their often-distressful routines may hamper the detection and handling of family violence cases. This study estimated the magnitude of violence against children reported by users of two emergency hospitals in Rio de Janeiro, Brazil. It also evaluated the degree of underreporting by contrasting the present findings to cases reported routinely. 524 parents/guardians of children under 12 treated at the hospitals from January to March 2005 were interviewed. Twelve-month prevalence of family violence was measured with the Conflict Tactics Scales: Parent-Child (CTSPC). All cases reported by staff and officially recorded in the preceding year were identified. Prevalence of psychological aggression, negligence, and physical violence were $94.8 \%$ (95\% CI: 92.9-96.2), 60.3\% (95\%CI: 55.9-64.7), and 47.2\% (95\%CI: 42.7-51.8), respectively. However, the corresponding prevalence rates according to routinely reported information were 0.007\% (95\%CI: 0.003-0.013), 0.24\% (95\%CI: 0.22-0.27), and 0.03\% (95\%CI: 0.02-0.04). This striking difference suggests that case identification and strategies to report violence against children in emergency rooms need to be reevaluated.

Child Abuse; Packaged Hospitals; Domestic Violence
Anna Tereza Miranda Soares de Moura ${ }^{1}$

Claudia Leite Moraes 2

Michael Eduardo Reichenheim 2

\section{Introdução}

A violência familiar contra a criança vem despertando crescente atenção nas últimas décadas, sendo reconhecida como um fenômeno freqüente e complexo a ser enfrentado no âmbito da saúde 1. Sua ocorrência resulta em conseqüências graves à saúde infantil, que podem se manifestar em curto, médio ou longo prazo 1,2,3. Em função de sua elevada magnitude e suas diferentes repercussões físicas, emocionais e sociais, a partir do final da década de 70 , a violência contra a criança passou a ser encarada como um grave problema de saúde pública 1 .

Nos países em desenvolvimento essa preocupação é um pouco mais recente. No Brasil, a partir de discussões envolvendo várias instâncias preocupadas com o bem-estar da criança, o problema vem sendo paulatinamente incorporado à agenda governamental, resultando em ações e políticas que objetivam a minimização de seus efeitos deletérios na saúde infantil 4,5,6. Muito se tem avançado até o momento e, desde 2001, os casos de maus-tratos contra a criança passaram a ser considerados como um agravo de notificação compulsória em todo o território nacional 4,5.

Conforme crescem as evidências sobre o impacto negativo da violência familiar no bem-estar da criança, cresce também o reconhecimento do papel dos serviços de educação e saúde em sua abordagem. Com base na convivência diária com as crianças, os professores são capazes de 
detectar alterações sutis de comportamento, que podem estar relacionadas à ocorrência de maustratos 7,8.

No âmbito da saúde, especial atenção vem sendo direcionada aos serviços de emergência, considerados como um local propício à suspeição, detecção e primeira abordagem das vítimas $9,10,11,12$. Durante os atendimentos podem surgir oportunidades privilegiadas para observação de lesões corporais agudas, tais como escoriações e fraturas sem motivos que as justifiquem, marcas de queimaduras propositais, dentre outros sinais considerados "típicos" de atos violentos. Ademais, diversos autores vêm chamando a atenção sobre a possibilidade de uma primeira intervenção das equipes em situações de estresse e conflito familiar 13,14. Os serviços de emergência oferecem assistência em plantões de 24 horas e um relativo anonimato durante o atendimento, este último podendo facilitar a revelação de situações de conflito. Representam ainda o primeiro e, por vezes, o único contato das vítimas com o setor de saúde 15. Mesmo quando a violência não é a queixa principal relatada por ocasião da consulta de emergência, a procura por assistência nestes serviços pode representar uma oportunidade de detecção de casos 10,16.

Apesar desses aspectos positivos, existem importantes lacunas na abordagem da violência familiar no âmbito dos serviços de emergência em todo o mundo. Alguns autores chegam a comentar que as estimativas de prevalência geradas nestes cenários correspondem tão somente ao nível de sensibilização e capacitação dos profissionais, e não à sua ocorrência real na clientela atendida 17 . A rotina agitada, a falta de tempo e privacidade, a ausência de recursos, bem como o pouco treinamento dos profissionais, são algumas particularidades apontadas como responsáveis por uma possível subdetecção de casos, o que gera uma casuística que acaba por representar apenas uma parcela do problema, o que favorece a perpetuação da violência e seu possível agravamento nos casos não identificados 9,18.

No Brasil, praticamente não existem estudos que avaliem a capacidade dos serviços de saúde, particularmente os de emergência, em detectar situações de maus-tratos contra a criança. Diante da importância dos serviços de emergência como uma das principais portas de entrada do setor da saúde e da escassez de informações sobre o tema nesses cenários, parece oportuno e relevante se debruçar sobre o tema. Baseando-se na avaliação da clientela de porta de entrada de dois grandes hospitais públicos de emergência do Rio de Janeiro, o estudo tem como objetivo estimar a magnitude da violência psicológica, violência física e negligência vivenciada por crianças que freqüentam estes serviços. Além da extensão do problema, pretende-se avaliar a capacidade dos profissionais para a detecção de casos de maustratos contra a criança, comparando a casuística notificada pelas equipes lotadas nos serviços de emergência com aquela estimada pela equipe da pesquisa com base em entrevistas nas salas de espera dos referidos hospitais. Os resultados poderão contribuir para o conhecimento mais abrangente da dinâmica de funcionamento nos serviços de emergência no que se refere à abordagem das crianças vítimas de maus-tratos, identificando potenciais problemas nas fases iniciais de suspeição, detecção e notificação de casos. Valendo-se dos conhecimentos gerados e de discussões cada vez mais ampliadas, propostas podem ser elaboradas para que as equipes lotadas nesses serviços possam melhor se adequar à difícil tarefa de encarar o desafio de proporcionar um enfrentamento apropriado às crianças vítimas.

\section{Métodos}

\section{Cenários de estudo}

Para compor a malha amostral, optou-se pela seleção de hospitais com atendimento de emergência pediátrica de grande porte (mais de 20 mil atendimentos pediátricos ao ano), localizados na Região Metropolitana do Rio de Janeiro, Brasil. Para que os cenários de atendimento representassem a clientela habitual dos serviços de emergência na região, foram excluídos os hospitais universitários, as maternidades e também aqueles com atendimento em subespecialidades médicas. Como critério de inclusão adicional, consideraram-se os hospitais com participação formal no Sistema de Notificação de Maus-Tratos Contra Crianças e Adolescentes do Estado do Rio de Janeiro, conforme recomendação do Ministério da Saúde 4,5. A malha final consistiu em cinco hospitais, dos quais foram selecionados, por conveniência, dois que representassem diferentes estágios de sensibilização profissional para a detecção e notificação das situações de maus-tratos contra a criança. O primeiro conta com equipe especificamente direcionada para abordagem dos casos de maus-tratos contra a criança e o segundo atua de acordo com as orientações gerais de notificar e encaminhar os casos detectados. 
Tamanho amostral e seleção de participantes

Para a busca ativa das situações de maus-tratos contra a criança foi realizado um estudo transversal envolvendo crianças que utilizaram os serviços de emergência entre janeiro e março de 2005. Foram realizadas 524 entrevistas face a face, distribuídas de forma semelhante entre os dois hospitais. Os critérios de inclusão levaram em consideração a idade da criança (1-12 anos incompletos) e a presença de acompanhantes que possuíssem vínculos de cuidado ou fossem seus principais responsáveis. As crianças que apresentavam quadro clínico muito grave que indicassem risco de vida foram excluídas da amostra. Tal decisão teve como objetivo não dificultar a prestação do primeiro atendimento à criança pela equipe da emergência e não importunar a família com uma temática potencialmente constrangedora em um momento de tamanho estresse. Além dessas, cinco entrevistas não foram consideradas nas análises por não preencherem os critérios anteriormente dispostos. O índice de recusa à participação foi de $3 \%$.

A captação dos sujeitos elegíveis, bem como as entrevistas ocorreram nas salas de triagem ou de espera dos hospitais. Após a identificação da respondente, esta era convidada a participar da pesquisa. As entrevistas eram realizadas antes ou após as consultas médicas, de acordo com o funcionamento de cada serviço, resguardando-se o anonimato e a privacidade das pessoas envolvidas. Ao término das entrevistas, todos os participantes receberam materiais instrutivos sobre prevenção de acidentes e violência familiar. Se observada a necessidade de acolhimento ou assistência, era oferecida à família a possibilidade de encaminhamento e orientação sobre onde procurar aconselhamento. A coleta de dados ocorreu ao longo dos sete dias da semana.

Instrumentos de coleta de dados para a

busca ativa de casos de maus-tratos

contra a criança

O questionário elaborado para a etapa de busca ativa continha em sua primeira parte questões referentes às características sócio-demográficas da clientela. Esse primeiro módulo tinha como objetivo estratégico estabelecer um vínculo entrevistador/entrevistado antes de se passar ao assunto principal da pesquisa. Para a identificação dos casos de maus-tratos contra a criança utilizou-se a Conflict Tactics Scales: Parent-Child (CTSPC) 19,20,21, formalmente adaptada para uso no Brasil 22,23. Voltado especificamente para identificar atos praticados pelos pais ou responsáveis legais, o instrumento foi elaborado no final da década de 90 como um desdobramento do instrumento anterior, amplamente validado e empregado em diferentes partes do mundo, utilizado para identificação de qualquer tipo de violência familiar (CTS1) 19,24. O módulo básico da CTSPC é composto por 22 itens divididos em três escalas: disciplina não-violenta (4 itens), agressão psicológica (5 itens) e agressão física (13 itens). Esta última é subdividida em três subescalas: punição corporal (5 itens), mau-trato físico (4 itens) e mau-trato físico grave (4 itens), sendo possível também o agrupamento das duas últimas subescalas, compondo-se a subescala de mau-trato físico total. O questionário ainda compreende três módulos sobressalentes que abordam as táticas utilizadas para disciplina infantil na semana anterior à entrevista (4 itens), situações de negligência (5 itens) e abuso sexual (4 itens). O instrumento foi aplicado em sua totalidade e apenas o módulo básico foi analisado neste estudo.

\section{Universo amostral e critérios de inclusão das fichas de notificação dos hospitais de emergência}

Para estimar a prevalência de casos de maustratos contra a criança notificados com base na própria rotina dos profissionais, todas as cópias das 932 fichas preenchidas pelos profissionais da equipe e encaminhadas aos Conselhos Tutelares nos 12 meses anteriores à entrevista em cada uma das unidades (ano de 2004) foram avaliadas na própria unidade, sendo incluídas no presente estudo apenas aquelas relativas a crianças menores de 12 anos atendidas nos serviços de emergência e que apresentassem um dos três tipos de maus-tratos contra a criança (agressão psicológica, física e/ou negligência) claramente definido. Um total de 440 fichas preencheu os critérios de inclusão acima detalhados.

\section{Análise de dados}

Para estimar a prevalência de maus-tratos contra a criança por meio da busca ativa de casos, consideraram-se como positivas as crianças que haviam sido vítimas de pelo menos um item das escalas de agressão psicológica, agressão física e negligência da CTSPC. Os respectivos intervalos de $95 \%$ de confiança (IC95\%) foram calculados via método binomial exato. A digitação, processamento e análise de dados foram realizados utilizando-se o programa Stata 9.0 (Stata Corp., College Station, Estados Unidos).

A prevalência de casos notificados foi obtida a partir da relação entre o número de fichas que reportavam os diferentes tipos de maus-tratos 
contra a criança e o número de atendimentos pediátricos realizados nos serviços de emergência durante os 12 meses que precederam o estudo (102.324 e 253.216 atendimentos nos hospitais 1 e 2, respectivamente).

Para fins de avaliação do grau de subnotificação das situações de violência nos serviços, comparam-se as estimativas oriundas das fichas de notificação com as do estudo transversal, assumindo-se que ambas cobrem a mesma experiência da clientela. Dessa forma, assume-se que as estimativas geradas a partir das notificações espontâneas geradas nos setores de emergência no ano anterior ao estudo (2004) representam as prevalências das diferentes formas de violência na população estudada, segundo a prática rotineira das equipes de emergência.

\section{Questões éticas}

O estudo foi aprovado pelo Comitê de Ética em Pesquisa da Secretaria Municipal de Saúde do Rio de Janeiro, em conformidade com os princípios da Declaração de Helsinki. A participação na pesquisa se seguiu à assinatura de Termo de Consentimento Livre e Esclarecido, que abordava os objetivos do estudo e as etapas metodológicas que previam a participação do respondente. Garantiu-se a confidencialidade das informações e o anonimato aos respondentes, e todos os casos positivos foram encaminhados para avaliação.

\section{Resultados}

O perfil da clientela que procurou os dois cenários de atendimento durante o período de estudo foi semelhante e está disposto na Tabela 1 . A maioria dos respondentes era mulher, casada, com mais de 25 anos, escolaridade inferior ao primeiro grau completo e do lar. Houve um ligeiro predomínio de crianças do sexo masculino. As famílias eram compostas, em sua maioria, por até quatro filhos, costumavam utilizar o serviço de emergência pelo menos quatro vezes ao ano e apresentavam renda mensal entre 1-4 salários mínimos da época. A média de idade foi de 30,8 anos (desvio-padrão - DP $=8,3$ ) para as respondentes e 5,1 anos ( $\mathrm{DP}=2,9)$ para as crianças.

As prevalências de maus-tratos contra a criança de acordo com a CTSPC encontram-se na Tabela 2. Observa-se um padrão decrescente na ocorrência dos atos violentos menos graves para os mais graves, independentemente se o perpetrador é o companheiro ou a mãe. Considerando a mãe como a perpetradora, chamam atenção as elevadas prevalências de agressão psicológica (94,8\%; IC95\%: 92,9-96,2) e castigo corporal (92,9; IC95\%: 90,8-94,6). As situações de negligência abarcam mais da metade da amostra (52,3\%; IC95\%: 48,7-55,9). Quanto à violência física, 38,7\% (IC95\%: 35,3-42,3) das entrevistadas relataram a prática de pelo menos um item da subescala de maus-tratos físicos, enquanto que 5,6\% (IC95\%: 4,1-7,5) revelaram ter praticado pelo menos um ato de maus-tratos físicos grave no ano anterior à entrevista. Os atos perpetrados pelo companheiro seguem esse mesmo padrão, porém são sistematicamente de menor magnitude. Ao focalizar os maus-tratos físicos como um todo, por exemplo, observou-se que a prevalência da violência perpetrada pelo companheiro estava abaixo da metade daquela perpetrada pela mãe.

Ao se considerar as prevalências referentes à ocorrência de maus-tratos perpetrados por pelo menos um membro do casal, as estimativas são ainda mais marcantes. A agressão psicológica e punição corporal passam a 96,5\% (IC95\%: 94,898,1) e 94\% (IC95\%: 91,5-95,9), respectivamente. A elevação das prevalências também atinge os demais tipos de violência. Os maus-tratos físicos, por exemplo, foram perpetrados contra cerca de 50\% (47,2\%; IC95\%: 42,7-51,8) das crianças do estudo, porquanto o mau-trato físico grave chega a quase $10 \%$ (8,2\%; IC95\%: 5,9-11,1) das mesmas.

O número de notificações bem como as prevalências estimadas por meio da avaliação das mesmas nos dois hospitais encontram-se dispostos na Tabela 3. Como pode ser observado, foram realizadas 440 notificações durante o período analisado. Ao se observar a distribuição das mesmas de acordo com o tipo de maus-tratos contra a criança, percebe-se um claro predomínio de notificações por suspeita de situações de negligência, já que este tipo de violência esteve presente em $85 \%$ das fichas. Apenas 12,5\% e 2,5\% das mesmas se referiram às violências física e psicológica, respectivamente. Quanto às prevalências estimadas, nota-se que estas foram baixas em todos os hospitais, embora tenham sido mais elevadas no segundo hospital em todos os tipos de maus-tratos contra a criança.

Comparando-se as prevalências estimadas pelas fichas de notificação às apresentadas na Tabela 2, oriundas da utilização da CTSPC, notase que as prevalências estimadas com base nas fichas de notificação são muito inferiores àquelas estimadas por meio do inquérito que utilizou a CTSPC. Enquanto a agressão psicológica foi reportada por 94,8\% (IC95\%: 92,9-96,2) das respondentes durante a busca ativa (Tabela 2), a prevalência estimada pelas fichas de notificação considerando-se os dois hospitais em conjunto foi de apenas 0,007\% (IC95\%: 0,003-0,013) (Tabela 3). Em relação aos maus-tratos físicos, en- 
Características da amostra de usuários dos serviços de emergência dos hospitais 1 e 2. Rio de Janeiro, Brasil, janeiro a março de 2005

\begin{tabular}{|c|c|c|}
\hline \multirow[t]{2}{*}{ Características da clientela } & \multicolumn{2}{|c|}{ Total $(\mathrm{N}=524)$} \\
\hline & $\%$ & IC95\% \\
\hline \multicolumn{3}{|l|}{ Idade da criança (anos) } \\
\hline Até 5 incompletos & 60,4 & $56,9-63,9$ \\
\hline 5-9 incompletos & 27,2 & $24,1-30,5$ \\
\hline 10-12 incompletos & 12,4 & $10,1-14,8$ \\
\hline \multicolumn{3}{|l|}{ Sexo } \\
\hline Feminino & 46,3 & $42,7-49,9$ \\
\hline Masculino & 53,7 & $50,0-57,3$ \\
\hline \multicolumn{3}{|l|}{ Idade da mãe (anos) } \\
\hline$<25$ & 24,2 & $21,3-27,4$ \\
\hline$>25$ & 75,8 & $72,6-78,7$ \\
\hline \multicolumn{3}{|l|}{ Número de filhos } \\
\hline Até 4 & 78,2 & $75,1-81,1$ \\
\hline Mais de 4 & 21,8 & $18,9-24,8$ \\
\hline \multicolumn{3}{|l|}{ Escolaridade da mãe } \\
\hline Até Ensino Fundamental incompleto * & 55,4 & $51,8-59,0$ \\
\hline De Ensino Fundamental completo até Ensino Médio incompleto ** & 28,0 & $24,8-31,3$ \\
\hline Ensino Médio completo ou mais & 16,6 & $14,0-19,4$ \\
\hline \multicolumn{3}{|l|}{ Trabalho da mãe } \\
\hline Do lar & 56,6 & $53,0-60,1$ \\
\hline Empregada & 43,4 & $39,9-47,0$ \\
\hline \multicolumn{3}{|l|}{ Situação conjugal } \\
\hline Solteira & 28,4 & $25,2-31,7$ \\
\hline Com companheiro & 71,6 & $68,3-74,8$ \\
\hline \multicolumn{3}{|l|}{ Trabalho do companheiro } \\
\hline Empregado & 84,8 & $81,6-87,7$ \\
\hline Desempregado & 15,2 & $12,3-18,4$ \\
\hline \multicolumn{3}{|l|}{ Freqüência ao setor de emergência (vezes ao ano) } \\
\hline Até 4 & 72,8 & $69,5-75,9$ \\
\hline Mais de 4 & 27,2 & $24,1-30,5$ \\
\hline \multicolumn{3}{|l|}{ Renda familiar mensal (salários mínimos) } \\
\hline Até 1 & 18,6 & $15,8-21,7$ \\
\hline $1-4$ & 73,4 & $70,0-76,7$ \\
\hline Acima de 5 & 8,0 & $6,0-10,2$ \\
\hline
\end{tabular}

* Ensino Fundamental corresponde a oito anos de estudo;

** Ensino Médio corresponde a três anos de estudo.

quanto a busca ativa revelou uma prevalência de 47,2\% (IC95\%: 42,7-51,8) na população estudada, a relativa às fichas de notificação foi de apenas 0,034\% (IC95\%: 0,025-0,045). Com relação à negligência, apesar do inquérito ter estimado uma prevalência de 60,3\% (IC95\%: 55,9-64,7), as fichas de notificação revelaram uma prevalência de apenas 0,24\% (IC95\%: 0,218-0,267).

\section{Discussão}

Este é o primeiro estudo a avaliar a ocorrência de maus-tratos contra a criança nos serviços de emergência de grandes hospitais públicos do Rio de Janeiro e a se debruçar sobre as possíveis oportunidades perdidas de detecção do evento nestes cenários. Em consonância com a literatura, os resultados da busca ativa de casos mostram que os diversos tipos de maus-tratos são bastante comuns entre crianças atendidas nos serviços de 
Prevalências de violência contra a criança perpetrada pela respondente e seu companheiro, com base na busca ativa de casos via entrevistas face a face. Rio de Janeiro, Brasil, janeiro a março de 2005

\begin{tabular}{|c|c|c|c|c|c|c|}
\hline \multirow[t]{2}{*}{ Tipos de maus-tratos } & \multicolumn{2}{|c|}{ Mãe (N = 540) } & \multicolumn{2}{|c|}{ Companheiro $(\mathrm{N}=347)$} & \multicolumn{2}{|c|}{$\begin{array}{l}\text { Pelo menos um dos } \\
\text { membros do casal } \\
\qquad(N=540)\end{array}$} \\
\hline & $n$ * & \% (IC95\%) & $n$ * & \% (IC95\%) & $n$ * & \% (IC95\%) \\
\hline Agressão psicológica & 512 & $94,8(92,9-96,2)$ & 267 & $77,1(73,4-80,9)$ & 521 & $96,5(94,8-98,1)$ \\
\hline Negligência & 282 & $52,3(48,7-55,9)$ & 142 & $44,0(39,5-48,6)$ & 326 & $60,3(55,9-64,7)$ \\
\hline Castigo corporal & 502 & $92,9(90,8-94,6)$ & 200 & $60,7(56,2-65,0)$ & 508 & $94,0(91,5-95,9)$ \\
\hline Maus-tratos físicos & 209 & $38,7(35,3-42,3)$ & 57 & $16,5(13,3-20,1)$ & 235 & $46,8(42,7-51,3)$ \\
\hline Maus-tratos físicos graves & 30 & $5,6(4,1-7,5)$ & 11 & $3,1(1,7-5,0)$ & 44 & $8,2(5,9-11,1)$ \\
\hline Maus-tratos físicos totais ** & 214 & $39,7(36,2-43,2)$ & 58 & $16,9(13,7-20,6)$ & 255 & $47,2(42,7-51,8)$ \\
\hline
\end{tabular}

* Total de casos positivos;

** Maus-tratos físicos totais incorporam a escala de maus-tratos físicos e maus-tratos físicos graves.

Tabela 3

Prevalências de violência contra a criança estimada com base nas fichas de notificação provenientes dos hospitais 1 e 2 no ano anterior à entrevista. Rio de Janeiro, Brasil.

\begin{tabular}{lcccccc}
\hline Tipos de maus-tratos & $\mathbf{n} *$ & $\begin{array}{c}\text { Hospital 1 } \\
\text { \% (IC95\%)** }\end{array}$ & $\mathbf{n} *$ & $\begin{array}{c}\text { Hospital 2 } \\
\%(\text { IC95\%) *** }\end{array}$ & $\begin{array}{c}\text { n * } \\
\%(I C 95 \%) \#\end{array}$ \\
\hline Agressão psicológica & 5 & $0,005(0,002-0,011)$ & 6 & $0,011(0,004-0,024)$ & 11 & $0,007(0,003-0,013)$ \\
Agressão física & 18 & $0,018(0,010-0,028)$ & 35 & $0,066(0,046-0,091)$ & 53 & $0,034(0,025-0,045)$ \\
Negligência & 160 & $0,156(0,133-0,182)$ & 216 & $0,406(0,354-0,464)$ & 376 & $0,242(0,218-0,267)$ \\
\hline
\end{tabular}

* Total de casos notificados no período estudado;

** Prevalência e seu intervalo de 95\% de confiança (IC95\%) calculados considerando-se o número de casos notificados no hospital 1 no numerador e o número de atendimentos de emergência relativo a esta faixa etária neste mesmo hospital no denominador (102.324 atendimentos);

*** Prevalência e seu IC95\% calculados considerando-se o número de casos notificados no hospital 2 no numerador e o número de atendimentos de emergência relativo a esta faixa etária neste mesmo hospital no denominador (53.216 atendimentos);

\# Prevalência e seu IC95\% calculados considerando-se a soma de casos notificados nos dois hospitais no numerador e o número de atendimentos de emergência relativo a esta faixa etária nos dois hospitais no denominador (155.540).

emergência, alcançando patamares superiores aos agravos mais freqüentemente identificados como motivo de procura às emergências, corroborando a idéia de que se trata de um problema relevante a ser enfrentado 18,25.

A subnotificação de casos encontrada no estudo se contrapõe, de certa forma, a algumas tendências internacionais que sugerem um número crescente de encaminhamento de casos para os serviços de proteção à criança nos últimos anos 26,27. Todavia, esse crescimento parece não acompanhar a magnitude de maus-tratos contra a criança e os números oficiais que se originam das notificações geradas nos serviços de saúde acabam por não representar a sua real ocorrência 12,28. Além da questão da baixa detecção, foco central deste estudo, outros fatores podem ser apontados como limitantes à prática da notificação. Dúvidas quanto aos encaminhamentos e condutas realizados pelos Conselhos Tutelares e outras instituições de proteção à criança, o despreparo das equipes e a falta de retorno de informações são apenas algumas das razões que dificultam a opção pela notificação 29 .

Especificamente no que tange à dificuldade para a detecção de casos, o fato parece estar relacionado a diferentes fatores. Alguns autores relatam que a subdetecção pode estar relacio- 
nada ao perfil do profissional envolvido com o atendimento 17 . Outros às circunstâncias que o envolvem ${ }^{30}$. No âmbito de uma equipe multiprofissional, o profissional teria de, além de observar e suspeitar, ser capaz de caracterizar o caso e optar por intervir, tarefas nem sempre conhecidas e claras. Por outro lado, existem algumas circunstâncias que parecem aumentar as chances de detecção e posterior notificação nos serviços de emergência, tais como a menor idade da vítima, a maior gravidade do caso, o fato de o perpetrador ser um desconhecido, a presença de ameaça de morte e de lesões ao exame físico 31 .

Como apresentado anteriormente, a Tabela 2 revela que a grande maioria das respondentes referiu uso de agressão psicológica contra as crianças atendidas no ano anterior à entrevista. Esses achados estão em consonância com a literatura que considera essa forma de agressão o tipo mais comum de maus-tratos contra a criança 1. Em contrapartida, essa forma de violência foi a menos notificada pelas equipes, sendo praticamente ignorada nos dois hospitais. É certo que os eventos que caracterizam a escala de agressão psicológica da CTSPC não costumam fazer parte das queixas que usualmente se apresentam nas salas de emergência. Muitos conflitos relatados pela clientela dificilmente seriam detectados nestes locais, cuja atuação é primordialmente centrada na resolução dos problemas agudos e urgentes apresentados pelos pacientes. Porém, como a agressão psicológica sofrida pela criança pode estar direta ou indiretamente envolvida na gênese do agravo que suscitou a consulta de emergência, esta forma de violência não deveria passar despercebida. Muitas vezes os próprios profissionais são testemunhas de situações que compõem essa escala, como o uso de ameaças e atitudes de depreciação direcionadas às crianças durante as consultas de emergência, o que já indicaria a necessidade de maior atenção ao caso e possível investigação mais aprofundada da situação.

Do mesmo modo, a opção por métodos de castigo corporal e pela agressão física como forma de resolução de conflito pela clientela foi bastante elevada. Quando se observa a ocorrência de maus-tratos físicos totais, somando-se os atos perpetrados pela mãe e pelo companheiro, é difícil encontrar na prática diária dos serviços de emergência um evento que apresente tamanha magnitude. Levando-se em conta que a ocorrência desses atos pode levar ao aparecimento das lesões físicas freqüentemente observadas nos serviços de emergência, é espantosa a baixa capacidade para a suspeição e detecção de casos por parte dos profissionais. Tal dificuldade não é exclusividade dos hospitais investigados. Pesqui- sas em países onde a discussão sobre a abordagem da violência contra a criança em serviços de saúde vem de mais longa data também indicam a pequena capacidade de identificar e notificar essas situações 15,32. Mesmo ao se contrastar os casos notificados de violência física (e, possivelmente, entendidos como de maior gravidade pelas equipes) com aqueles detectados mediante a ocorrência de pelo menos um dos itens da escala de maus-tratos físicos graves na CTSPC ("bater muito, sem parar, até o máximo possível"; "agarrar pelo pescoço e sacudir"; "queimar ou escaldar" e/ou "ameaçar/usar faca ou arma"), a diferença ainda é muito marcante. A enorme magnitude da subnotificação de situações dessa natureza impõe que se reflita sobre as estratégias que estão sendo utilizadas na sensibilização das equipes de emergência, na medida em que se está diante de situações hediondas e que absolutamente não poderiam deixar de ser suspeitadas para posterior investigação.

A magnitude das situações de negligência também chama a atenção. O problema foi detectado pela busca ativa de casos em cerca de $50 \%$ da clientela, com a freqüência dos atos perpetrados pelo pai e pela mãe alcançando patamares semelhantes. Porém, enquanto esse tipo de maus-tratos contra a criança não foi o mais identificado por meio da busca ativa, foi aquele que mais motivou os profissionais de saúde a preencherem uma ficha de notificação. Como mostra a Tabela 3, o número de notificações de negligência foi cerca de seis vezes maior do que a soma de todas as outras fichas de notificação ao se considerar os dois hospitais em conjunto. Apesar de ser o tipo de maus-tratos contra a criança mais notificado, ainda existe uma grande discrepância entre as duas fontes de informação, indicando que várias destas situações também não vêm sendo identificadas.

Analisando-se o perfil das situações classificadas como negligência segundo a CTSPC e as motivações descritas nas fichas para a notificação de casos, é possível postular que as duas estratégias estejam identificando crianças diferentes como vítimas. As fichas de notificação classificadas com esse tipo de maus-tratos contra a criança traziam, na sua maioria, relatos de situações de conflito entre os profissionais atendentes e as famílias, tais como a retirada do paciente à revelia, uma discordância com o atendimento proposto e agressões à equipe de atendimento. Além de serem distintas aos itens que compõem a escala do instrumento, as justificativas oferecidas não fazem parte da definição habitualmente utilizada para as situações de negligência 1,5. Essa desconexão pode ser decorrente da complexidade e dificuldade inerente à definição desse tipo 
de maus-tratos contra a criança, principalmente em países em desenvolvimento, onde é difícil separar a negligência do Estado da praticada pelos responsáveis pela criança ${ }^{33}$. Avaliações acerca do conteúdo dos encaminhamentos estão em curso, o que permitirá conhecer melhor o que realmente chama atenção do profissional lotado nos serviços de emergência e o que o faz desencadear uma notificação.

Os achados do estudo devem ser avaliados à luz de suas limitações. A primeira diz respeito às estimativas da ocorrência de maus-tratos contra a criança na clientela usuária dos serviços de emergência. Estas podem estar subestimadas, já que não foram contemplados atos perpetrados em outras relações familiares além dos pais e ou responsáveis legais da criança. A exclusão das crianças graves do estudo também pode ter contribuído para subestimação das situações de violência. Tal decisão metodológica se sustentou em alguns aspectos éticos que merecem comentário. O primeiro é que em se tratando de um caso grave e de emergência, a entrevista com os responsáveis poderia dificultar a prestação de esclarecimentos por parte da família sobre o quadro clínico da criança, prejudicando o seu atendimento pela equipe. Em segundo lugar, pensou-se que a abordagem da família sobre um tema difícil como o da violência doméstica poderia ser inadequada nessas situações, na medida em que poderia mobilizar sentimentos de culpa e apreensão, trazendo um estresse ainda maior à família, já transtornada pelo risco de vida da criança. Imaginou-se ainda que seria difícil localizar os responsáveis por crianças internadas nas salas de repouso e unidades de terapia intensiva, visto que a permanência dos responsáveis nestes setores ainda é restrita em alguns hospitais.

Ademais, ainda que o instrumento de aferição utilizado para a identificação das situações de violência (CTSPC) tenha um excelente histórico psicométrico prévio 19,20,21, é possível que alguns atos de maus-tratos contra a criança não tenham sido revelados por serem estes fortemente desaprovados socialmente. A subidentificação de algumas situações mais graves se torna ainda mais plausível, considerando-se que as entrevistas foram realizadas nas salas de espera e/ou triagem dos hospitais, o que fez com que fosse difícil garantir a privacidade desejada em algumas situações. Como o assunto focalizado é visto como constrangedor e íntimo, a sua abordagem nesses cenários poderia ser considerada inapropriada pelos pacientes e seus familiares, levando a um menor relato da sua ocorrência.

Outro ponto importante diz respeito à restrição do estudo aos maus-tratos psicológicos, físicos e negligência, deixando de fora as situações de abuso sexual. Tal decisão se justificou pela falta de um espaço exclusivo para as entrevistas, pelo pouco tempo que se teve para as mesmas em função da dinâmica atribulada dos serviços de emergência, pela ausência de informações sobre a adequação da CTSPC como estratégia de identificação de casos dessa forma de abuso no contexto dos serviços de saúde e porque, diferentemente das outras escalas, a escala de abuso sexual da CTSPC não se restringe à investigação dos abusos cometidos pelos pais ou responsáveis, foco central da pesquisa. Seria interessante que estudos futuros abordassem o tema, já que a identificação, o registro e a notificação de casos de abuso sexual contra a criança têm características próprias, não permitindo a extrapolação dos achados deste estudo para tais situações.

Uma questão concernente à validade da estratégia utilizada com vistas à avaliação do nível de subnotificação do problema também merece reflexão. Para que se possa aceitar a adequação da comparação entre as estimativas oriundas de duas fontes (notificação e busca ativa) é necessário assumir que ambas se reportam ao mesmo domínio de estudo e que, efetivamente, cubram a mesma experiência da clientela. Devese aceitar que, além da população se encontrar em estabilidade, as duas fontes de informação se referem à mesma janela temporal. Nesse sentido, há de se admitir que as informações oriundas das notificações não se confinam apenas a situações de violência pontuais e recentes, mas àquelas que vêm ocorrendo há algum tempo. No limite, o período a ser assumido deve ser de 12 meses, uma vez que esta é a janela temporal de recordatório utilizada nas aferições de eventos via CTSPC. A pertinência da comparação pode ser reforçada pelo fato da casuística da busca ativa ser, em princípio, representativa da clientela atendida nos hospitais. Por outro lado, não é possível resgatar o período (médio) ao qual os eventos notificados se reportam. Somente um estudo específico poderia responder a essa questão e, possivelmente, corroborar a vinculação das duas fontes de dados. Por ora, as inferências requerem certo cuidado.

O estudo também apresenta pontos positivos e inovadores que merecem destaque. Os resultados observados trazem uma visão diferenciada da ocorrência de maus-tratos contra a criança em hospitais de emergência. A maioria das informações presente na literatura se baseia primordialmente na análise de situações graves, aquelas relacionadas a lesões corporais, ou ainda, de casos já detectados e em acompanhamento nos serviços de proteção à criança 9,16,34,35. Destaca-se aqui, a abordagem de uma clientela espontânea, sem a escolha prévia de grupos populacionais 
específicos, tipos de queixas ou de pacientes. Essa opção permite uma visão mais abrangente do problema, já que a magnitude de maus-tratos contra a criança foi detectada na população que freqüentemente utiliza os serviços de emergência e que pode representar os usuários dos serviços de saúde em geral. Essa assertiva tem um peso ainda maior em países em desenvolvimento como o Brasil, onde existe grande dificuldade na organização da porta de entrada dos sistemas públicos de saúde.

Ao evidenciar um descompasso entre as respostas da clientela e os casos encaminhados pelas equipes de emergência, ressalta-se a importância da capacitação dos profissionais envolvidos com o problema. Como já mencionado, o destaque dos serviços de emergência em todo esse processo é fundamental e inquestionável. Suspeitar e detectar "sinais de alerta" nesses locais significa dar conta de uma clientela que freqüentemente não possui acesso a outros recursos da rede. As equipes lotadas nos serviços de emergência precisam estar sensibilizadas e envolvidas com o processo de suspeição, detecção e notificação para que as casuísticas dos serviços possam ser estimadas de forma mais fidedigna e abrangente. Outro ponto interessante revelado pelo estudo é o predomínio da notificação de alguns tipos de maus-tratos contra a criança em detrimento de outros. O entendimento das motivações que levam as equipes a iniciar o processo de notificação merece ser aprofundado, para que se possa atuar exatamente nas especificidades de cada serviço e nas dificuldades percebidas pelos profissionais envolvidos com $\mathrm{o}$ atendimento.

A necessidade de aprimorar as estratégias de capacitação e organização dos serviços de emergência também fica evidente ao se considerar que algumas etapas na abordagem das vítimas de maus-tratos contra a criança são próprias destes serviços. É o caso, por exemplo, da contracepção de emergência e profilaxias das DST/AIDS, recomendadas em casos de abuso sexual. Muitos autores sugerem que a ocorrência de maus-tratos contra a criança seja rotineiramente investigada pelas equipes dos serviços de emergência $36,37,38$. Porém, ainda não existe consenso em como lidar com essas situações, principalmente em serviços onde a rotina é bastante atribulada e estressante. A forma ideal e o momento adequado para realizar essa difícil tarefa ainda é um desafio para os profissionais de saúde preocupados com o problema 18,39 .

Além dos obstáculos relativos à organização dos serviços de emergência, vários são os entraves que dificultam a incorporação da abordagem de maus-tratos contra a criança em suas rotinas. No Brasil, como em outros países em desenvolvimento, a implementação de políticas muitas vezes esbarra na carência de recursos e na ausência de programas de capacitação e educação profissional continuada. Além disso, as recomendações referentes ao manejo das situações de maus-tratos contra a criança ainda não estão adequadamente disseminadas, alcançando as unidades da rede de saúde de maneira desigual. Ainda é possível observar a superposição de atividades referentes à abordagem das vítimas de violência nos serviços envolvidos com essa rede de atenção, além da dificuldade ou até a ausência de retorno quando os casos são encaminhados. Esses problemas já vêm sendo apontados na literatura como possíveis entraves à notificação de casos pelas equipes de saúde 40 .

\section{Conclusão}

As prevalências detectadas por meio da busca ativa de casos na clientela atendida foram bastante altas e expressivamente superiores às estimadas com base nas fichas de notificação encaminhadas pelas equipes de plantão. Mesmo considerando apenas os casos mais graves e de apresentação mais exuberante, a diferença continua expressiva, indicando uma real subnotificação por parte dos profissionais, o que recomenda a revisão das estratégias que vêm sendo utilizadas no treinamento das equipes, uma melhor organização dos serviços para o enfrentamento do problema e uma maior integração do setor saúde com as demais instâncias de proteção à criança. O reconhecimento de que se trata de uma questão grave, freqüente e merecedora de atenção especial pode ser um passo inicial para uma abordagem empática e acolhedora das crianças vítimas de violência familiar que chegam às emergências. 


\section{Resumo}

O enfrentamento da violência contra a criança é considerado um desafio nos serviços de emergência, onde a rotina atribulada pode dificultar a detecção dos casos. O presente estudo estimou a magnitude da violência contra crianças atendidas em dois hospitais de emergência no Rio de Janeiro, Brasil. Também avaliou o grau de sub-registro de casos, comparando a casuística notificada pelas equipes com aquela estimada pelo estudo. Para aferição da violência foi utilizado o instrumento Conflict Tactics Scales: Parent-Child (CTSPC), aplicado em 524 acompanhantes de crianças atendidas nos hospitais entre janeiro e março de 2005. Foram avaliadas todas as notificações originadas da identificação de casos pelas equipes em 2004. De acordo com a CTSPC, a prevalência de violência psicológica, negligência e violência física foi de 94,8\% (IC95\%: 92,996,2), 60,3\% (IC95\%: 55,9-64,7) e 47,2\% (IC95\%: 42,751,8), respectivamente. Já estas estimativas segundo as notificações foram de 0,007\% (IC95\%: 0,003-0,013), 0,24\% (IC95\%: 0,22-0,27) e 0,03\% (IC95\%: 0,02-0,04). Essa considerável diferença entre as estimativas do estudo e as relativas aos casos notificados sugere que as estratégias de identificação e notificação de casos de violência contra a criança nos serviços de emergência sejam reavaliadas.

Maus-Tratos Infantis; Hospitais de Emergência; Violência Doméstica

\section{Referências}

1. Krug EG, Dahlberg LL, Mercy JA, Zwi AB, Lozano R. World report on violence and health. Geneva: World Health Organization; 2002.

2. Diaz A, Simantov E, Rickert VI. Effect of abuse on health: results of a national survey. Arch Pediatr Adolesc Med 2002; 156:811-7.

3. Pinheiro P. World report on violence against children. Geneva: United Nations Secretary-General's Study on Violence against Children; 2006.

4. Ministério da Saúde. Política nacional de redução da morbimortalidade por acidentes e violências. Brasília: Ministério da Saúde; 2001.

5. Ministério da Saúde. Notificação de maus-tratos contra crianças e adolescentes pelos profissionais de saúde: um passo a mais na cidadania em saúde. Brasília: Ministério da Saúde; 2002.

6. Minayo MCS. A difícil e lenta entrada da violência na agenda do setor saúde. Cad Saúde Pública 2004; 20:646-7.

7. Webster S, O'Toole R, O'Toole A, Lucal B. Overreporting and underreporting of child abuse: teachers' use of professional discretion. Child Abuse Negl 2005; 29:1281-96.

\section{Colaboradores}

A. T. M. S. Moura participou das seguintes etapas: escolha do tema, organização do trabalho, coleta de dados, supervisão do trabalho de campo e digitação dos dados, análise dos dados, discussão, organização do artigo e edição final do texto. M. E. Reichenheim e C. L. Moraes colaboraram nas etapas: escolha do tema, organização do trabalho, análise dos dados, discussão, organização e correção do artigo, revisão e edição final do texto.
8. Kenny M. Teachers' attitudes toward and knowledge of child maltreatment. Child Abuse Negl 2004; 28:1311-9.

9. Ziegler DS, Sammut J, Piper AC. Assessment and follow-up of suspected child abuse in preschool children with fractures seen in a general hospital emergency department. J Paediatr Child Health 2005; 41:251-5.

10. Keshavarz R, Kawashima R, Low C. Child abuse and neglect presentations to a pediatric emergency department. J Emerg Med 2002; 23:341-5.

11. Stuart P. Risk factors for recent domestic physical assault in patients presenting to the emergency department. Emerg Med Australas 2004; 16: 216-24.

12. MacMillan HL, Jamieson E, Walsh CA. Reported contact with child protection services among those reporting child physical and sexual abuse: results from a community survey. Child Abuse Negl 2003; 27:1397-408

13. Spivey M, Schnitzer P, Kruse R, Slusher P, Jaffe D. Association of injury visits in children and child maltreatment reports. J Emerg Med; no prelo. 
14. Krugman SD, Lantz PE, Sinal S, De Jong AR, Coffman K. Forced suffocation of infants with baby wipes: a previously undescribed form of child abuse. Child Abuse Negl 2007; 31:615-21.

15. Sanders T, Cobley C. Identifying non-accidental injury in children presenting to A\&E departments: an overview of the literature. Accid Emerg Nurs 2005; 13:130-6.

16. Benger JR, Pearce V. Simple intervention to improve detection of child abuse in emergency departments. BMJ 2002; 324:780.

17. Gough D. Defining the problem. Child Abuse Negl 1996; 20:993-1002.

18. King W, Reid C. National audit of emergency department child protection procedures. Emerg Med J 2003; 20:222-4.

19. Straus M, Hamby S, Finkelhor D, Moore D, Runyan D. Identification of child maltreatment with parent-child conflict tactics scales: development and psychometric data for a national sample of American parents. Child Abuse Negl 1998; 22:249-70.

20. de Zoysa P, Rajapakse L, Newcombe PA. Adaptation and validation of the parent-child conflict tactics scale for use in Sri Lanka. Ceylon Med J 2005; 50:11-4.

21. Bennett DS, Sullivan MW, Lewis M. Relations of parental report and observation of parenting to maltreatment history. Child Maltreat 2006; 11: 63-75.

22. Reichenheim ME, Moraes CL. Adaptação transcultural do instrumento Parent-Child Conflict Tactics Scales (CTSPC) utilizado para identificar a violência contra a criança. Cad Saúde Pública 2003; 19:1701-12.

23. Reichenheim ME, Moraes CL. Psychometric properties of the Portuguese version of the Conflict Tactics Scales: Parent-child Version (CTSPC) used to identify child abuse. Cad Saúde Pública 2006; 22:503-15.

24. Straus MA, Hamby SL, Boney-McCoy S, Sugarman DB. The revised Conflict Tactics Scales (CTS2): development and preliminary psychometric data. J Fam Issues 1996; 17:283-316.

25. Sidebotham P, Biu T, Goldsworthy L. Child protection procedures in emergency departments. Emerg Med J 2007; 24:831-5.

26. Delaronde S, King G, Bendel R, Reece R. Opinions among mandated reporters toward child maltreatment reporting policies. Child Abuse Negl 2000; 24:901-10.
27. Feng JY, Levine M. Factors associated with nurses' intention to report child abuse: a national survey of Taiwanese nurses. Child Abuse Negl 2005; 29:783-95.

28. Flaherty EG, Sege R, Binns HJ, Mattson CL, Christoffel KK. Health care providers' experience reporting child abuse in the primary care setting. Pediatric Practice Research Group. Arch Pediatr Adolesc Med 2000; 154:489-93.

29. Melton GB. Mandated reporting: a policy without reason. Child Abuse Negl 2005; 29:9-18.

30. Ashton V. The effect of personal characteristics on reporting child maltreatment. Child Abuse Negl 2004; 28:985-97.

31. Finkelhor D, Ormrod RK. Factors in the underreporting of crimes against juveniles. Child Maltreat 2001; 6:219-29.

32. Dowd D, Bull M. Emergency medicine and injury prevention: meeting at the intersection. Clin Pediatr Emerg Med 2003; 4:83-9.

33. Straus MA, Kantor GK. Definition and measurement of neglectful behavior: some principles and guidelines. Child Abuse Negl 2005; 29:19-29.

34. Fletcher AK, Burke DP. Presentation to accident and emergency with crying or screaming and likelihood of child protection registration. Emerg Med J 2002; 19:17-8.

35. Fung EL, Sung RY, Nelson EA, Poon WS. Unexplained subdural hematoma in young children: is it always child abuse? Pediatr Int 2002; 44:37-42.

36. Morrison LJ, Allan R, Grunfeld A. Improving the emergency department detection rate of domestic violence using direct questioning. J Emerg Med 2000; 19:117-24.

37. Krasnoff M, Moscati R. Domestic violence screening and referral can be effective. Ann Emerg Med 2002; 40:485-92.

38. Boyle A, Robinson S, Atkinson P. Domestic violence in emergency medicine patients. Emerg Med J 2004; 21:9-13.

39. Sheehan K. Intentional injury and violence prevention. Clin Pediatr Emerg Med 2003; 4:12-20.

40. Wright RJ, Wright RO, Farnan L, Isaac NE. Response to child abuse in the pediatric emergency department: need for continued education. Pediatr Emerg Care 1999; 15:376-82.

Recebido em 02/Out/2007

Versão final reapresentada em 19/Mai/2008 Aprovado em 26/Mai/2008 